\title{
RESISTÊNCIA AO INTEMPERISMO ARTIFICIAL DE CINCO MADEIRAS TROPICAIS E DE DOIS PRODUTOS DE ACABAMENTO
}

\section{RESISTANCE TO ARTIFICIAL WEATHERING OF FIVE TROPICAL WOODS AND OF TWO FINISH PRODUCTS}

\author{
Janine Oliveira e Silva ${ }^{1}$ Tereza Cristina Monteiro Pastore ${ }^{2}$ Floriano Pastore Junior ${ }^{3}$
}

\section{RESUMO}

Os objetivos do trabalho foram avaliar o processo de fotodecomposição da madeira de cinco espécies tropicais e analisar o efeito de proteção de dois produtos de acabamento de madeiras quando submetidos ao intemperismo artificial. Foram empregadas as madeiras de ipê-roxo (Tabebuia impeginosa), itaúba (Mezilaurus itauba), maçaranduba (Manilkara huberi), tatajuba (Bagassa guianensis) e tauari (Couratari sp.). Amostras destas madeiras foram submetidas a ciclos de radiação ultravioleta (UV) e água até perfazer um total de 2.000 horas de irradiação e 400 horas de lixiviação em água. A mudança da cor natural das madeiras, indicativo do processo de fotodegradação, e o grau de proteção de um verniz poliuretânico transparente e de um "stain" semitransparente foram monitorados por espectrocolorimetria. As espécies mais resistentes foram o ipê-roxo e tauari, seguidas pela maçaranduba, itaúba e tatajuba respectivamente. O verniz forneceu proteção por tempo maior ao ipê-roxo e à maçaranduba, enquanto o "stain" foi mais eficiente para a itaúba e tatajuba. Ambos os produtos tiveram desempenho similar para a madeira de tauari.

Palavras-chave: Fotodegradação da madeira; sistema CIE-L*a*b*; produtos de acabamento.

\section{ABSTRACT}

This work aimed at evaluating the photodecomposition process of five tropical wood species and the protection effect of two wood finishing products under artificial weathering. Woods of Tabebuia impeginosa, Mezilaurus itauba, Manilkara huberi, Bagassa guianensis and Couratari sp. were employed. Samples were submitted to cycles of 2,000 hours of ultraviolet radiation (UV) and 400 hours of water leaching. Changes in the natural color of wood, indicative of the photodegradation process, and the protection degree of a transparent poliuretanic varnish and a semitransparent stain were monitored by spectrocolorimetry. The most resistant wood species were Tabebuia impetiginosa and Couratari sp. followed by Manilkara huberi, Mezilaurus itauba and Bagassa guianensis, respectively. The varnish protected Tabebuia impetiginosa and Manilkara huberi for a long period, while the stain seemed to be more efficient for Mezilaurus itauba and Bagassa guianensis. Both products presented similar performance for Couratari sp.

Keywords: Wood photodegradation; CIE-L*a*b* system; finishing products.

\section{INTRODUÇÃO}

A durabilidade natural ou resistência à degradação é definida como a capacidade do cerne da madeira de qualquer espécie florestal resistir à ação de agentes deterioradores (Eaton e Hale, 1993) e, atualmente, abrange tanto os agentes biológicos quanto os físicos e químicos (Zabel e Morrell, 1992). No entanto, em função dos maiores graus de ocorrência e importância, muitas vezes, o termo resistência natural é indevidamente utilizado, restringindo-se à ação de microorganismos.

Para reduzir o período de duração e os custos dos testes em campo, para a avaliação do intemperismo, foram desenvolvidos ensaios de laboratório que simulam o intemperismo e permitem estimar e comparar a durabilidade natural das madeiras de diversas espécies (Oliveira et al, 1986).

A madeira submetida a intempéries, em curto período de tempo, apresenta variação de cor, posteriormente, a superfície torna-se áspera, provocada pela erosão causada pela água da chuva, que remove

1. Bacharel em Química, Instituto de Química, Universidade de Brasília, CEP 70904-970, Brasília (DF).

2. Bacharel em Química, $\operatorname{Dr}^{\mathrm{a}}$., Pesquisadora do Laboratório de Produtos Florestais, Instituto Brasileiro do Meio Ambiente, SCEN Trecho 02, Edifício Sede do IBAMA, CEP 70818-900, Brasília (DF). tereza.pastore@ibama.gov.br

3. Bacharel em Química, MSc., Professor Assistente do Instituto de Química, Universidade de Brasília, CEP 70904970, Brasília (DF).

Recebido para publicação em 25/04/2006 e aceito em 6/11/2006. 
os polímeros decompostos e deteriora a microestrutura (Daniel et al., 2004). O intemperismo, ação complexa e combinada do sol, da chuva ou umidade e dos ventos, é um processo superficial iniciado pela luz solar, que penetra apenas 2,0 mm abaixo da superfície (Hon, 2001). A radiação ultravioleta - UV $(30-400 \mathrm{~nm})$ do espectro solar é o fator considerado mais prejudicial, pois desencadeia reações de decomposição química da celulose, lignina e hemiceluloses (Feist e Hon, 1984).

Apesar da ação do intemperismo ser limitada à superfície da madeira, constitui um problema para os usuários e para os tecnologistas, porque afeta o desempenho dos produtos aplicados sobre ela. Por exemplo, as resinas que contêm compostos absorvedores de UV possuem tempo de vida limitado, exigem manutenção freqüente, o que inclui a sua remoção total e preparação de nova superfície (Feist e Hon, 1984). Além disso, os produtos semitransparentes permitem que a radiação atinja a superfície da madeira, degradando-a antes mesmo do revestimento (Pandey e Pitman, 2002; Silva e Pastore, 2004) e reduzindo o grau de adesão entre o produto e substrato (Pandey e Pitman, 2002).

Os compostos químicos cromóforos que causam variação da cor da madeira submetida ao intemperismo foram estudados por Hon (2001), tendo a colorimetria de refletância difusa sido a técnica mais usada para acompanhar essas mudanças (Castellan et al., 1996; Greleier et al., 1997; Camargos e Gonçalez, 2001; Gonçalez et al., 2001; Nzokou e Kamdem, 2002; Pastore et al., 2004; Silva e Pastore, 2004).

Os objetivos do trabalho foram avaliar o processo de fotodecomposição da madeira de cinco espécies tropicais e analisar o efeito de proteção de dois produtos de acabamento de madeiras quando submetidos ao intemperismo artificial.

\section{MATERIAL E MÉTODOS}

$\mathrm{Na}$ pesquisa, foram utilizados discos das madeiras, coletados na base do tronco comercial das árvores, de ipê-roxo (Tabebuia impetiginosa (Mart. Ex DC. Standl.), itaúba (Mezilaurus itauba (Meisn.) Taub. Ex Mez), maçaranduba (Manilkara huberi (Ducke) Chevalier), tatajuba (Bagassa guianensis Aubl.) e tauari (Couratari sp.), provenientes da Floresta Nacional do Tapajós (PA), que se encontravam armazenados no Laboratório de Produtos Florestais - LPF/IBAMA/Brasília. Essas espécies tropicais foram selecionadas por serem tradicionalmente indicadas para uso exterior (IBDF,1983; Souza, 1997). Por essa razão, é importante conhecer o comportamento dessas madeiras quando expostas ao intemperismo e à extensão da degradação dessas espécies, que não estão bem documentados e precisam ser investigados.

Nove amostras medindo $5,0 \times 2,0 \times 0,3 \mathrm{~cm}$ foram retiradas do cerne do mesmo disco, em seqüência da casca para a medula, sendo o comprimento no sentido longitudinal, a largura no sentido tangencial e a espessura no sentido radial. As superfícies de maiores dimensões da amostra, a que será tratada com radiação UV e a superfície oposta a ela foram aplainadas e polidas com lixas.

Seguindo a tendência atual de manter a beleza natural e, ao mesmo tempo, proteger a madeira (Daniel et al., 2004), os produtos de acabamento selecionados foram o verniz poliuretânico transparente e o "stain" semitransparente. Apesar de possuírem mecanismos de proteção diferentes, ambos possuem duplo filtro solar, ou seja, compostos que absorvem a energia UV, dissipando-a em forma de calor e, assim, reduzindo a velocidade de degradação da superfície.

Das nove amostras de cada espécie, três serviram como testemunha (sem acabamento), três foram tratadas com verniz poliuretânico (transparente brilhante) e três com "stain" (semitransparente) e, seguindo as instruções dos fabricantes, foram aplicadas três demãos. Nas composições desses produtos, conforme descrito na embalagem, são incluídos pigmentos inorgânicos que refletem a radiação UV e fungicidas e bactericidas para combater o ataque de microorganismos.

O detalhamento do procedimento de simulação de intemperismo por meio de ciclos de 10 horas de irradiação UV, seguido por 2 horas de lixiviação e secagem em temperatura ambiente (média de três dias) encontra-se descrito em Silva e Pastore (2004). O tempo total de irradiação foi 2.000 horas e o de lixiviação foi 400 horas.

As medidas da cor foram feitas no espectrofotômetro "Datacolor International Microflash 200D" como descrito por Silva e Pastore (2004). O espectro final representa o valor médio de nove medições realizadas em pontos diferentes da superfície das três amostras. As diferenças entre os parâmetros 
colorimétricos do sistema CIE-L*a*b* de 1976 (Billmeyer e Satzman, 1981) foram usadas para quantificar a mudança total de cor $\Delta \mathrm{E}^{*}$ por meio da Equação 1.

$$
\Delta \mathrm{E}^{*}=\left(\Delta \mathrm{L}^{* 2}+\Delta \mathrm{a}^{* 2}+\Delta \mathrm{b}^{* 2}\right)^{1 / 2}
$$

Em que: $\Delta \mathrm{L}^{*}=$ variação da Luminosidade (amostra tratada menos testemunha); $\Delta \mathrm{a}^{*} \mathrm{e} \Delta \mathrm{b}^{*}=$ variação das Coordenadas cromáticas (amostra tratada menos testemunha).

Os sinais positivo e negativo fornecidos pelo sistema significam: $+\Delta \mathrm{L}^{*}$ aumento da luminosidade; $-\Delta \mathrm{L}^{*}$ redução da luminosidade; $+\Delta \mathrm{a}^{*}$ aumento na cor vermelha; $-\Delta \mathrm{a}^{*}$ aumento na cor verde; $+\Delta \mathrm{b}^{*}$ aumento na cor amarela $\mathrm{e}-\Delta \mathrm{b}^{*}$ aumento na cor azul.

\section{RESULTADOS E DISCUSSÃO}

A Figura 1A ilustra os espectros de refletância difusa no visível das madeiras de ipê-roxo, itaúba, maçaranduba, tatajuba e tauari. Esses espectros representam as cores descritas pelo sistema Munsell (Souza, 1997) que estão relacionadas Tabela 1.

A aplicação do verniz ou do "stain" escureceu todas as madeiras estudadas, conforme o exemplo do tauari mostrado na Figura 1B, pois ocorreu uma diminuição na intensidade da luz refletida. Também é possível observar nessas duas curvas que, na região entre 540 - $550 \mathrm{~nm}$, ocorreu uma inflexão, o que sugere a presença de algum pigmento ou aditivo orgânico nos produtos de acabamento utilizados. Em geral, esses compostos são adicionados para reduzir a degradação da madeira por meio de reflexão ou absorção da luz UV (Daniel et al., 2004), mesmo que os produtos sejam transparentes (verniz) e semitransparentes ("stain").

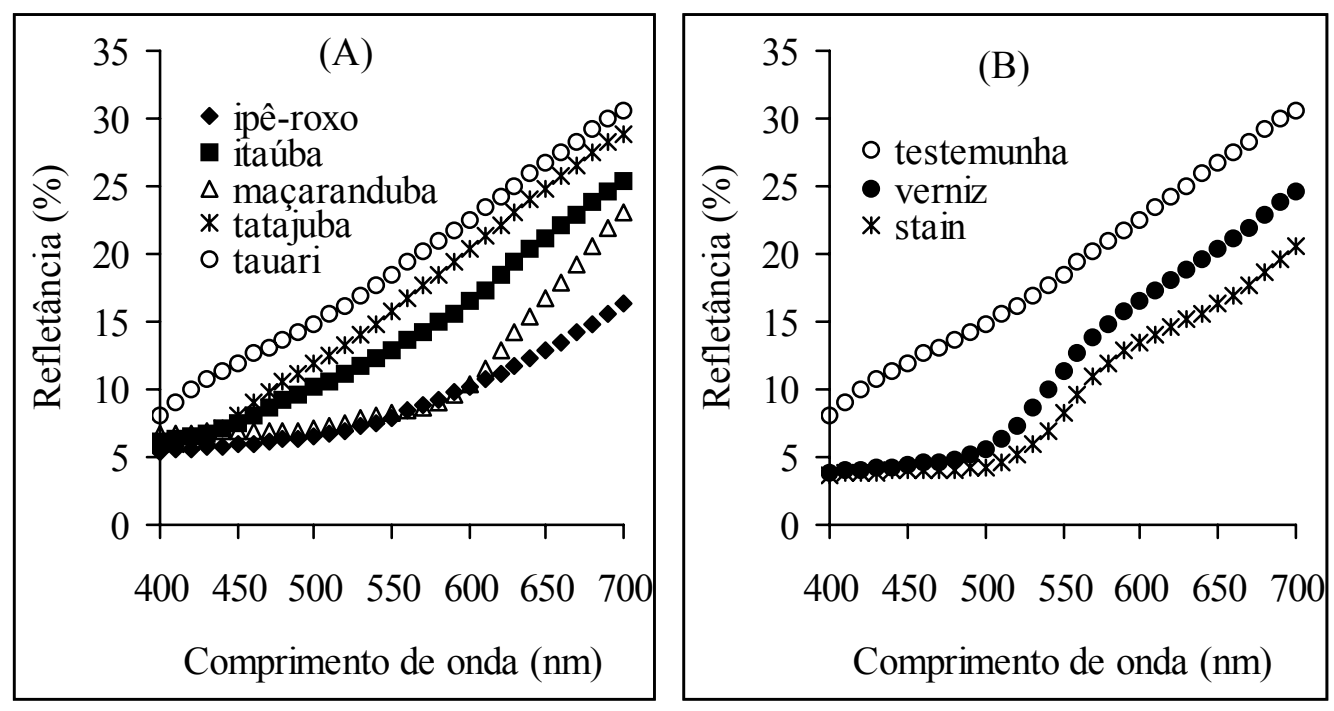

FIGURA 1: Espectro da cor natural do ipê-roxo, itaúba, maçaranduba, tatajuba e tauari (A) e amostras de tauari testemunha e tratadas com verniz e "stain" (B).

FIGURE 1: Natural colors curves of Tabebuia impetiginosa, Mezilaurus itauba, Manilkara huberi, Bagassa guianensis and Couratari sp. woods (A), and control and samples covered with varnish and stain of tauari wood (B).

Os dados das cores das espécies antes da exposição UV são apresentados na Tabela 1. Pelos valores da luminosidade $\left(\mathrm{L}^{*}\right)$, as espécies estudadas podem ser classificadas, grosseiramente, como madeiras claras (tatajuba e tauari), escuras (ipê-roxo e maçaranduba), ficando a itaúba entre esses extremos. Essa classificação pode ser visualizada pela observação da ordem de disposição dos espectros na Figura $1 \mathrm{~A}$, em que, as madeiras claras refletem mais radiação e seus espectros encontram-se acima das curvas das madeiras escuras. 
TABELA 1: Parâmetros da cor natural das amostras.

TABLE 1: Color parameters of unexposed samples.

\begin{tabular}{l|cc|c|l}
\hline Espécie & $\mathrm{L}^{*(1)}$ & $\mathrm{a}^{*(2)}$ & $\mathrm{b}^{*(3)}$ & \multicolumn{1}{c}{ Cor $^{(4)}$} \\
\hline Ipê-roxo & 34,67 & 6,71 & 9,15 & Oliva-amarronzado-escuro \\
Itaúba & 43,22 & 6,74 & 16,88 & Marrom-amarelado \\
Maçaranduba & 35,74 & 8,64 & 7,27 & Marrom-avermelhado-escuro \\
Tatajuba & 47,09 & 6,76 & 21,27 & Marrom-dourado \\
Tauari & 50,43 & 5,72 & 15,65 & Marrom-amarelado-claro \\
\hline
\end{tabular}

Em que: $\mathrm{L}^{*}=$ luminosidade; $\mathrm{a}^{*}=$ coordenada cromática verde-vermelho; $\mathrm{b}^{*}=$ coordenada cromática amarelo-azul; Conforme o sistema Munsell de ordenamento de cor (Souza, 1997).

As variações dos parâmetros colorimétricos provocadas pelos ciclos de radiação UV e lixiviação das madeiras encontram-se na Tabela 2. Inicialmente, os ciclos de tratamento provocaram redução na luminosidade ou escurecimento $\left(-\Delta \mathrm{L}^{*}\right)$ da superfície de todas as amostras. Verifica-se o comportamento inverso, após um período dependente da espécie estudada, que variou de 200 a 600 horas de irradiação. A luminosidade começa a aumentar, indicando o início do processo de clareamento. Estudos feitos com madeiras de clima temperado (Muller et al., 2003) ou tropicais (Nzokou e Kamdem, 2002) relatam esse comportamento. $\mathrm{O}$ escurecimento inicial é atribuído a formação de compostos quinoidais em função, sobretudo, da degradação da lignina pela radiação UV na presença de oxigênio (Hon, 2001). Esses compostos são lixiviados pela água, deixando a madeira mais áspera e esbranquiçada, por causa do aumento do teor de celulose que, conseqüentemente, causa aumento da luminosidade. Das espécies estudadas, a itaúba $\left(\Delta \mathrm{L}^{*}=-8,81\right)$ e a tatajuba $\left(\Delta \mathrm{L}^{*}=-6,79\right)$ foram aquelas que apresentaram maior modificação da luminosidade.

TABELA 2: Variações dos parâmetros de cor das superfícies in natura das madeiras.

TABLE 2: Change in color parameters of in natura surfaces of woods.

\begin{tabular}{|c|c|c|c|c|c|c|c|c|c|c|c|}
\hline \multirow{2}{*}{ Parâmetro } & \multirow{2}{*}{ Espécie } & \multicolumn{10}{|c|}{ Tempo de exposição (h) } \\
\hline & & 10 & 20 & 50 & 100 & 200 & 400 & 600 & 1000 & 1500 & 2000 \\
\hline \multirow{5}{*}{$\Delta \mathrm{L}^{*(1)}$} & Ipê roxo & $-1,72$ & $-1,14$ & $-1,64$ & $-1,63$ & $-2,70$ & $-2,38$ & $-1,89$ & $-1,03$ & 0,41 & 1,49 \\
\hline & Itaúba & $-5,65$ & $-6,36$ & $-7,88$ & $-7,81$ & $-9,42$ & $-10,35$ & $-10,32$ & $-10,83$ & $-9,53$ & $-8,81$ \\
\hline & Maçaranduba & $-5,66$ & $-6,42$ & $-7,52$ & $-7,35$ & $-7,39$ & $-6,88$ & $-5,91$ & $-4,98$ & $-2,88$ & $-1,04$ \\
\hline & Tatajuba & $-4,84$ & $-6,95$ & $-9,23$ & $-11,10$ & $-11,21$ & $-12,16$ & $-13,03$ & $-11,39$ & $-8,84$ & $-6,79$ \\
\hline & Tauari & $-1,54$ & $-1,46$ & $-2,79$ & $-2,90$ & $-2,90$ & $-3,92$ & $-3,60$ & $-1,79$ & $-0,37$ & 0,78 \\
\hline \multirow{5}{*}{$\Delta \mathrm{a}^{*(2)}$} & Ipê roxo & 0,61 & 0,63 & 0,27 & 0,19 & 0,00 & $-0,58$ & $-0,91$ & $-1,26$ & $-1,66$ & $-2,06$ \\
\hline & Itaúba & 2,81 & 3,05 & 2,79 & 2,45 & 1,74 & 0,81 & 0,36 & 0,33 & $-0,63$ & $-1,10$ \\
\hline & Maçaranduba & 2,00 & 1,27 & 0,47 & $-0,41$ & $-1,61$ & $-3,04$ & $-3,87$ & $-4,42$ & $-5,00$ & $-5,28$ \\
\hline & Tatajuba & 3,31 & 4,47 & 4,34 & 3,67 & 2,93 & 1,25 & 0,67 & $-0,05$ & $-1,12$ & $-2,04$ \\
\hline & Tauari & 1,01 & 1,20 & 1,31 & 1,21 & 1,19 & 0,62 & 0,16 & $-0,46$ & $-0,96$ & $-1,46$ \\
\hline \multirow{5}{*}{$\Delta \mathrm{b}^{*(3)}$} & Ipê roxo & 1,84 & 2,14 & 1,20 & 0,74 & $-0,28$ & $-1,02$ & $-1,40$ & $-1,37$ & $-1,47$ & $-1,50$ \\
\hline & Itaúba & 0,10 & $-0,64$ & $-2,81$ & $-4,23$ & $-6,15$ & $-7,75$ & $-8,66$ & $-8,58$ & $-9,28$ & $-9,67$ \\
\hline & Maçaranduba & 1,71 & 0,81 & $-0,59$ & $-1,58$ & $-2,50$ & $-3,19$ & $-3,52$ & $-3,32$ & $-3,04$ & $-2,75$ \\
\hline & Tatajuba & 1,15 & $-0,87$ & $-3,95$ & $-6,88$ & $-8,93$ & $-11,22$ & $-12,23$ & $-12,22$ & $-12,76$ & $-13,75$ \\
\hline & Tauari & 3,10 & 3,78 & 3,68 & 3,51 & 2,95 & 1,30 & 0,06 & $-0,89$ & $-1,91$ & $-3,30$ \\
\hline \multirow{5}{*}{$\Delta \mathrm{E}^{*(4)}$} & Ipê roxo & 2,60 & 2,51 & 2,05 & 1,80 & 2,71 & 2,66 & 2,52 & 2,13 & 2,25 & 2,95 \\
\hline & Itaúba & 6,32 & 7,08 & 8,81 & 9,21 & 11,38 & 12,96 & 13,48 & 13,82 & 13,31 & 13,13 \\
\hline & Maçaranduba & 6,24 & 6,59 & 7,56 & 7,53 & 7,97 & 8,17 & 7,89 & 7,44 & 6,52 & 6,04 \\
\hline & Tatajuba & 5,97 & 8,31 & 10,94 & 13,57 & 14,63 & 16,59 & 17,89 & 16,71 & 15,57 & 15,47 \\
\hline & Tauari & 3,61 & 4,23 & 4,80 & 4,71 & 4,30 & 4,17 & 3,61 & 2,06 & 2,17 & 3,69 \\
\hline
\end{tabular}

Em que: $\Delta \mathrm{L}^{*}=$ variação da luminosidade; $\Delta \mathrm{a}^{*}=$ variação na coordenada cromática verde-vermelha; $\Delta \mathrm{b}^{*}=$ variação na coordenada cromática amarela-azul; $\Delta \mathrm{E}^{*}$ = variação total da coloração.

Os valores da coordenada $\Delta \mathrm{a}^{*}$ (Tabela 2) decresceram gradativamente durante o tratamento de intemperismo simulado, o que significa diminuição da concentração de compostos cromóforos de cor vermelha. Após 1.000 horas, todas as madeiras estudadas, exceto a itaúba, apresentaram valores negativos de $\Delta \mathrm{a}^{*}$. Nas condições desse ensaio, a maçaranduba sofreu a maior mudança na cor vermelha $\left(\Delta \mathrm{a}^{*}=-5,28\right)$ e a 
itaúba $\left(\Delta \mathrm{a}^{*}=-1,10\right)$ e o tauari $\left(\Delta \mathrm{a}^{*}=-1,46\right)$ as menores variações, portanto, essas últimas espécies apresentaram maior estabilidade em relação ao vermelho.

A evolução da formação e lixiviação dos compostos de cor amarela durante o tratamento pode ser acompanhada pelos valores da coordenada $\Delta \mathrm{b}^{*}$ relacionados na Tabela 2.

O comportamento da itaúba, maçaranduba e tatajuba foram semelhantes durante as primeiras 20 horas de tratamento, ou seja, a quantidade de pigmentos amarelos formados foi menor do que os lixiviados e ocorreu diminuição progressiva nos valores de $\Delta b^{*}$. Já as madeiras de ipê-roxo e tauari apresentaram aumento inicial na quantidade de compostos cromóforos amarelados nas primeiras 20 horas de intemperismo, para em seguida, iniciarem a redução.

É oportuno lembrar que madeiras submetidas somente à irradiação UV intensificam continuamente a cor amarela (Pastore, 2004). Portanto, no caso do tauari cujos valores de $\Delta \mathrm{b}^{*}$ passaram a ser negativos somente depois de $600 \mathrm{~h}$ de irradiação UV e $120 \mathrm{~h}$ de lixiviação, cabe observar que os cromóforos amarelos formados apresentam solubilidade em água muito mais baixa do que os formados nas outras madeiras estudadas (Tabela 2).

Na Figura 2, estão ilustradas as curvas das variações das coordenadas colorimétricas das testemunhas do tauari (A) e da tatajuba (B) que mostram a tendência de ambas as espécies à coloração azul com o aumento do número de ciclos de intemperismo artificial. A itaúba (não-ilustrada) também apresentou essa mesma tendência, que pode ser interpretada como a remoção dos cromóforos de cor amarela.

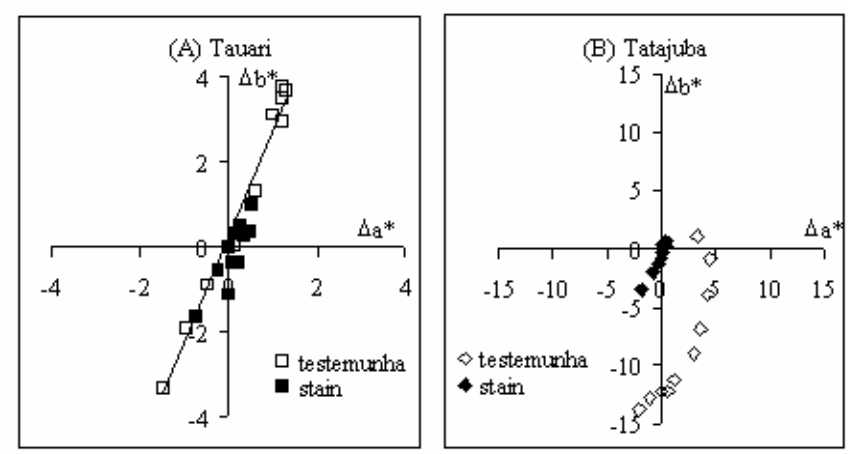

FIGURA 2: Curvas de variação de cor $\Delta \mathrm{a}^{*}$ versus $\Delta \mathrm{b}^{*}$ provocada pelo intemperismo artificial do tauari (A) e da tatajuba (B) com e sem "stain".

FIGURE 2: Curves of color variation $\Delta \mathrm{a}^{*}$ versus $\Delta \mathrm{b}^{*}$ due to artificial weathering woods of Couratari sp (A) and Bagassa guianensis (B) with and without stain.

Por outro lado, a tendência da maçaranduba e do ipê foi em direção à coloração verde, como mostrado nas Figuras 3A e 3B, indicando que pigmentos vermelhos estão sendo removidos da superfície exposta pelo processo de intemperismo artificial.
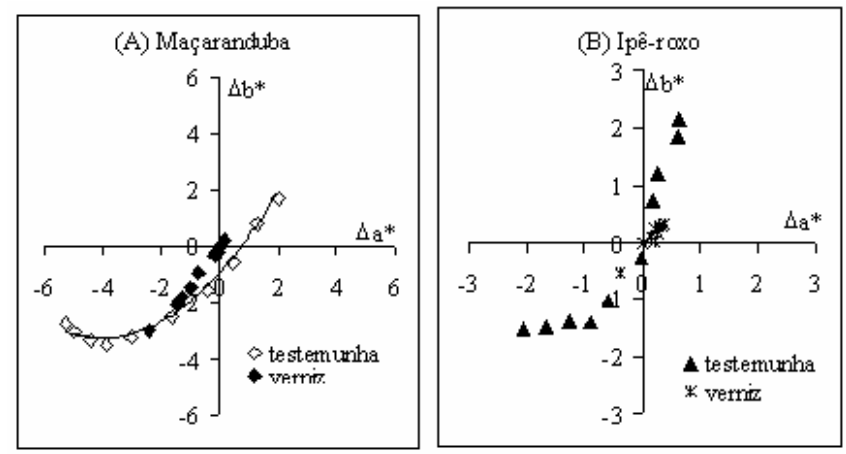

FIGURA 3: Curvas de variação de cor $\Delta \mathrm{a}^{*}$ versus $\Delta \mathrm{b}^{*}$ causadas pelo intemperismo artificial de madeiras de maçaranduba (A) e ipê-roxo (B) com e sem verniz.

FIGURE 3: Curves of color variation of $\Delta \mathrm{a}^{*}$ versus $\Delta \mathrm{b}^{*}$ due to artificial weathering of Manilkara huberi (A) and Tabebuia impetiginosa (B) woods with and without varnish. 
Pelo valor de $\Delta \mathrm{E}^{*}=13,13$ (Tabela 2 ) a itaúba sob intemperismo comportou-se como a tatajuba, uma madeira de cor clara com $\Delta \mathrm{E}^{*}=15,47$, apesar de não ter sido considerada clara pelos dados relacionados na Tabela 1. Os fatores que mais contribuíram para esse valor elevado foram as variações da luminosidade $\left(\Delta \mathrm{L}^{*}=-8,8\right)$ e da coordenada cromática amarela $\left(\Delta \mathrm{b}^{*}=-9,67\right)$. Na Figura 4 , esse comportamento da itaúba torna-se evidente.

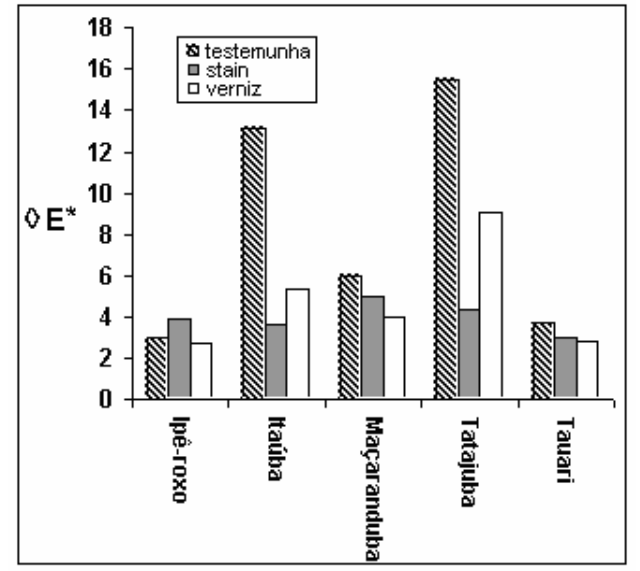

FIGURA 4: Modificação total da cor $\left(\Delta \mathrm{E}^{*}\right)$ das cinco madeiras estudadas, testemunhas e amostras protegidas com "stain" e verniz, após $2.000 \mathrm{~h}$ de radiação UV e $400 \mathrm{~h}$ de lixiviação.

FIGURE 4: Total color modifications $\left(\Delta \mathrm{E}^{*}\right)$ of the five studied woods, controls and samples protected with "stain" and varnish, after 2,000 h of UV irradiation and $400 \mathrm{~h}$ of lixiviation.

Outra madeira que apresentou comportamento atípico foi o tauari. Apesar de ser a madeira de cor mais clara $\left(\Delta \mathrm{L}^{*}=50,43\right)$ entre as estudadas (Tabela 1), teve o desempenho medido pela variação total de cor muito próximo ao do ipê-roxo, $\Delta \mathrm{E}^{*}=3,69$ e $\Delta \mathrm{E}^{*}=2,95$ respectivamente. Portanto, foi a segunda madeira mais resistente do grupo estudado. As causas desse desempenho estão sendo objeto de estudo no Laboratório de Produtos Florestais - IBAMA.

A variação total de cor das amostras-testemunha ou recobertas com produtos de proteção pode ser monitorada e quantificada pelo espectrocolorímetro, como mostrado na Figura 4. Percebe-se facilmente que as madeiras naturalmente menos resistentes são a tatajuba e a itaúba e que os dois produtos de acabamento aplicados sobre elas, reduziram em cerca de $50 \%$ a velocidade de degradação das superfícies. Nas condições experimentais estabelecidas, o "stain" semitransparente foi o produto mais resistente para essas duas espécies. O desempenho do "stain" aplicado sobre a tatajuba está ilustrado na Figura 2B e pode ser comparado com a atuação da mesma madeira sem proteção, por meio das extensões das duas curvas.

As espécies com maior resistência natural ao intemperismo foram ipê-roxo, tauari e maçaranduba respectivamente. Para esse grupo de madeiras, a eficiência dos produtos de acabamento não ficou tão evidenciada como no caso da itaúba e da tatajuba. Porém, percebe-se que o verniz poliuretânico foi o produto que apresentou proteção ligeiramente melhor que o "stain" para o ipê-roxo e maçaranduba. Para o tauari, os dois produtos em estudo tiveram desempenhos semelhantes.

Curiosamente, as resistências às intempéries da maçaranduba e da tatajuba (Souza, 1997) encontramse descritas na literatura como boa e baixa respectivamente, porém ao serem rastreadas, essas informações parecem advir do conhecimento popular. Este trabalho confirmou tais dados.

Vale lembrar que a comparação direta dos resultados deste trabalho com o desempenho das mesmas madeiras recobertas ou não com os produtos de acabamento sob intemperismo natural não é procedente, uma vez que para simular a lixiviação os corpos-de-prova foram totalmente imersos em água.

\section{CONCLUSÕES}

A espectrocolorimetria mostrou-se uma ferramenta adequada para monitorar o processo de fotodegradação das madeiras por 2.000 horas, em função da variação de cor ocasionada pela decomposição da lignina e dos extrativos sobretudo. 
Nas condições experimentais usadas, o ipê-roxo, tauari e maçaranduba apresentaram maior resistência natural à degradação por intemperismo simulado. As madeiras menos resistentes foram a itaúba e tatajuba.

O verniz poliuretânico forneceu proteção ligeiramente maior que o "stain" para as madeiras de ipê e maçaranduba. Por sua vez, o "stain" foi mais eficiente para a tatajuba e itaúba. Os dois produtos apresentaram proteção semelhante para o tauari

\section{AGRADECIMENTOS}

Ao Laboratório de Materiais e Combustíveis do Instituto de Química da Universidade de Brasília, pelo uso do reator UV e ao Conselho Nacional de Desenvolvimento Científico e Tecnológico (CNPq) pela bolsa de Iniciação Científica.

\section{REFERÊNCIAS BIBIOGRÁFICAS}

BILLMEYER JUNIOR, F.W.; SATZMAN, M. Principles of colour technology. New York: John Wiley, 1981. 240 p.

CAMARGOS, J.A.A.; GONÇALEZ, J.C. A colorimetria aplicada como instrumento na elaboração de uma tabela de cores de madeira. Brasil Florestal, Brasília, ano 20, n. 71, p. 30-41, 2001.

CASTELLAN, A.; NOURMAMOUDE, A.; GRELIER, S.; et al.. Photocoloration du bois de pin maritime em presénce d'additifs protecteurs. Cellulose Chem. Technol., Bucareste, v. 30, n. 5-6, p. 431-452, 1996.

DANIEL, T.; HIRSCH, M. S.; MCCLELLAND, K.; et al.. Finding the balance between aesthetics and durability. J. C. T. Coating Tech., Blue Bell, v. 1, n. 9, p. 42-48, 2004.

EATON, R.A.; HALE, M.D.C. Wood: decay, pests, and protection. Cambridge: Chapman \& Hall, 1993. 541 p.

FEIST, W. C.; HON, D. N. S. Chemistry of weathering e protection. In: ROWELL, R.M. The chemistry of solid wood. Washington: Am. Chem. Soc, 1984. 614 p.

GONÇALEZ, J.C.; JANIN, G.; SANTORO, A.C.S.; et al. Colorimetria quantitativa: uma técnica objetiva de determinar a cor da madeira. Brasil Florestal, Brasília, ano 20, v. 72, p. 47-58, 2001.

GRELEIER, S.; CASTELLAN, A.; DESROUSSEAUX, S.; NOURMAMODE; L. PODGORSKI, A. Attempt to protect wood colour against UV visible light by using antioxidants bearing isocyanate groups grafted to the material with microwave. Holzforschung, Berlin, v. 51, n. 6, p. 511-518, 1997.

HON, D.N.-S. Weathering and photochemistry of wood. In: Hon, D.N.-S.; Shiraishi, N. Wood and cellulosic chemistry. 2.ed. New York: Marcel Dekker, 2001. 928 p.

instituto BRASileiro DE DeSenVolvimento florestal. Potencial madeireiro do Grande Carajás. Brasília: IBDF/LPF, 1983. $134 \mathrm{p}$.

MÜLLER, U.; RÄTZSCH, M.; SCHWANNINGER, M.; et al.. Yellowing and IR-changes of spruce wood as result of UV-irradiation. J. Photochem. Photobiol. B: Biol., Lausanne, v. 69, n. 2, p. 97-105, 2003.

NZOKOU, P.; KAMDEM, D.P. Weathering of two hardwoods species: african paudak (Pterocarpus soyauxii) and red maple (Acer rubum). J. of Tropical Forest Products, Kuala Lumpur, v. 8, n. 2, p. 200-209, 2002.

OLIVEIRA, A.M.F.; LELIS, A.T.; LEPAGE, E.S.; et al. Agentes destruidores da madeira. In: Lepage, E.S. Manual de preservação de madeiras. São Paulo: IPT, 1986. v. 1, p. 99-278.

PANDEY, K. K.; PITMAN, A. J. FTIR studies of the changes in wood chemistry following decay by brown-rot and white-rot fungi. International Biodeterioration \& Biodegradation. Birmingham, v. 52, n. 3, p. 151-160, 2002.

PASTORE, T.C.M. Estudos do efeito da radiação ultravioleta em madeiras por espectroscopias Raman (FTRaman), de refletância difusa no infravermelho (DRIFT) e no visível (CIE-L*a*b*). 2004. 117f. Tese (Doutorado em Química Analítica) - Instituto de Química, Universidade de Brasília, Brasília, 2004.

PASTORE, T.C.M.; SANTOS, K.O.; RUBIM, J.C. A spectrometric study on the effect of ultraviolet irradiation of four tropical hardwoods. Bioresource Technology, Essex, v. 93, n. 1, p. 37-42, 2004.

SILVA, J.O.; PASTORE, T.C.M. Fotodecomposição e proteção de madeiras tropicais. Floresta e Ambiente, Seropédica, v. 21, n. 2, p. 7-13, 2004.

SOUZA, M. H. Madeiras tropicais brasileiras. Brasília: IBAMA/LPF, 1997. 152p.

ZABEL, R.A.; MORRELL, J.J. Wood microbiology: decay and its prevention. San Diego: Academic Press, 1992. 476p. 